\title{
The Boomerang: A Crushed and Re-born PWN?
}

\author{
Roland Kothes ${ }^{1}$, Bülent Uyanıker ${ }^{2} \&$ Wolfgang Reich ${ }^{2}$ \\ ${ }^{1}$ National Research Council of Canada, Herzberg Institute of \\ Astrophysics, Dominion Radio Astrophysical Observatory, P.O. Box \\ 248, Penticton, British Columbia, V2A 6J9, Canada \\ ${ }^{2}$ Max-Planck-Institut für Radioastronomie, Auf dem Hügel 69, 53121 \\ Bonn, Germany
}

\begin{abstract}
We present new high radio frequency observations of the Boomerang pulsar wind nebula (PWN) made with the Effelsberg 100-m radio telescope. A comparison with low frequency data from the Canadian Galactic Plane Survey (CGPS; Taylor et al. 2003) reveals a change of the nebula's emission structure with frequency caused by a radial steepening of the radio spectrum above $5 \mathrm{GHz}$. We also find evidence that the reverse shock of the initial supernova shock wave has driven away or crushed the original PWN which might explain why the current nebula around the pulsar has such a low radio luminosity.
\end{abstract}

\section{Introduction}

The Galactic supernova remnant (SNR) G106.3+2.7 is located within a large $\mathrm{HI}$ bubble at a distance of about $800 \mathrm{pc}$. A small arc-like synchrotron nebula, the "Boomerang", powered by the energetic pulsar PSR J2229+6114 with a rotational energy loss rate $\dot{E}=2.2 \times 10^{37} \mathrm{ergs} \mathrm{s}^{-1}$ (Halpern et al. 2001), is located at the top of the SNR's head (Fig. 1). Kothes, Uyaniker \& Pineault (2001) suggest that the progenitor star, as a member of the Cepheus OB2 association, exploded at the present location of the pulsar. To the northeast (NE), the shock wave has been quickly decelerated against dense material. Towards the south it has expanded into a moderately dense medium creating the head structure of the SNR. The shock wave is currently breaking out in the western direction into a region of much lower density. Its age is between 3000 and 10,000 years.

\section{Results}

During the summer of 2002 we observed the Boomerang with the Effelsberg $100-\mathrm{m}$ radio telescope at $4.85 \mathrm{GHz}, 8.35 \mathrm{GHz}$, and $10.45 \mathrm{GHz}$. A comparison of the new high frequency observations with the $1420 \mathrm{MHz}$ data of the CGPS reveals that (i) the Boomerang shows a break at $5 \mathrm{GHz}$ (Fig. 1), (ii) its structure changes with frequency - indicating a radial steepening of the spectrum, (iii) the radio luminosity is about a factor of 100 too low for such a pulsar in contrast 
to its X-ray emission which is in the expected range, and (iv) there is diffuse radio emission between the $\mathrm{HI}$ gas in the east and a $\mathrm{CO}$ filament in the west.
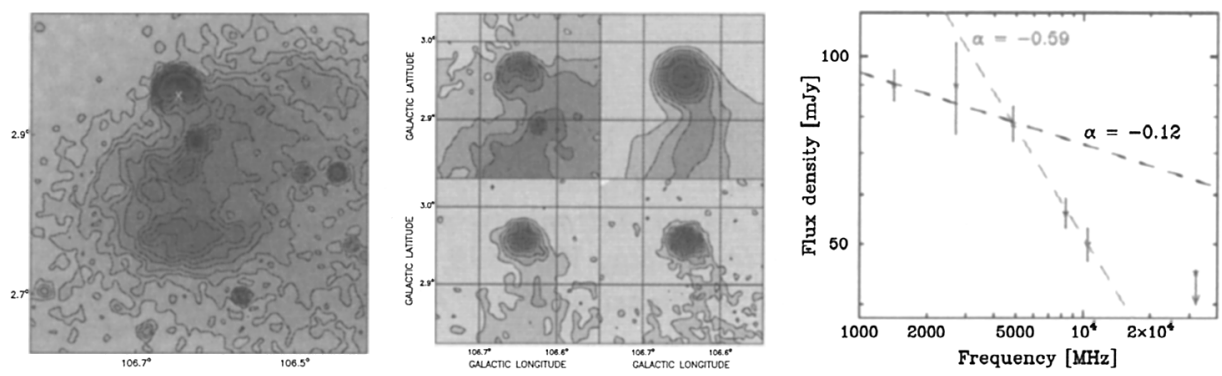

Figure 1. $1420 \mathrm{MHz}$ radio images of the SNR's head (left) and the Boomerang (middle, upper left). The new observations of the Boomerang at $4.85 \mathrm{GHz}, 8.35 \mathrm{GHz}$, and $10.45 \mathrm{GHz}$ are shown in the middle panel (upper right, lower left, and lower right, respectively). The radio spectrum of the Boomerang is shown on the right.

The evolution of G106.3+2.7 affects the emission structure of the shell-type SNR and also that of the PWN. As shown by Blondin et al. (2001) for the Vela SNR, a density gradient can lead to an offset position of the pulsar from the center of the PWN due to an asymmetric reverse shock. This should be observable in radio emission but not necessarily in X-rays since the lifetimes of X-ray-emitting electrons are shorter than their radio counterparts'. Compared to Vela, the Boomerang is an extreme case because the explosion took place next to an HI wall to the NE. The reverse shock from this direction quickly traveled back and pushed the nebula in the opposite direction. This explains both the low radio intensity of the Boomerang and the bright smooth radio emission filling the void between the HI gas in the east and molecular gas in the west.

Since we observe a flat spectrum at the position of the pulsar, with a radial steepening, the $5 \mathrm{GHz}$ break cannot be due to a break in the injected electron spectrum, but is the result of synchrotron cooling. For a cooling break at such a low frequency we need a magnetic field strength of a few mG. Assuming a symmetric wind from the pulsar, a radius of $0.3 \mathrm{pc}$ for the Boomerang, equipartition in energy between particles and magnetic field, and a pure dipole field for the pulsar, we can estimate the time the pulsar needs to generate enough energy to create $\mathrm{mG}$ fields from its $\dot{E}$. In this case the reverse shock should have passed the pulsar merely $360 \mathrm{yr}$ ago for a field of $1 \mathrm{mG}$ or $5000 \mathrm{yr}$ ago for $5 \mathrm{mG}$.

\section{References}

Blondin, J. M., Chevalier, R. A., \& Frierson, D. M. 2001, ApJ, 563, 806

Halpern, J. P., Camilo, F., Gotthelf, E. V., Helfand, D. J., Kramer, M., Lyne, A. G., Leighly, K. M., \& Eracleous, M. 2001, ApJ, 552, L125

Kothes, R., Uyanıer, B., \& Pineault, S. 2001, ApJ, 560, 236

Taylor, A. R., et al. 2003, AJ, 125, 3145 